\title{
MECP2 gene mutation analysis in Chinese patients with Rett syndrome
}

\author{
Hong Pan $^{1,4}$, Yan-Ping Wang ${ }^{2,3,4}$, Xing-Hua Bao ${ }^{1}$, Hong-Di Meng ${ }^{1}$, Yan Zhang ${ }^{2,3}, X$-Ru Wu ${ }^{1}$ \\ and Yan Shen*,2,3 \\ ${ }^{1}$ Department of Pediatrics, First Hospital of Peking University, Beijing, 100034, China; ${ }^{2}$ Institute of Basic Medical \\ Sciences, CAMS \& PUMC, Beijing, 100005, China; ${ }^{3}$ Chinese National Human Genome Center, Beijing, 100176, \\ China
}

Rett syndrome (RTT) is a progressive neurodevelopmental disorder that affects almost exclusively girls. Mutations in the X-linked methyl-CpG-binding protein 2 gene (MECP2) have been found to be a cause. In order to study the spectrum of MECP2 mutations in Chinese patients, we employed PCR and sequencing of the coding region of MECP2 gene in 31 Chinese cases of classical sporadic RTT. Mutations in MECP2 were found in about 55\%. Twelve different mutations in exon 3 were identified in 17 of these 31 patients; two of these are novel. A novel missense variant was detected in the C-terminal region in a patient and her father who was normal. In addition, there was a single nucleotide variant in the $3^{\prime}$ UTR. European Journal of Human Genetics (2002) 10, 484-486. doi:10.1038/sj.ejhg.5200827

Keywords: Rett syndrome; MECP2 gene; mutation

\section{Introduction}

Rett syndrome (RTT, MIM 312750) is a progressive neurodevelopmental disorder that affects girls almost exclusively, with an estimated prevalence of approximately 1 in 10000 - 15000 females.

Recently Amir et $a l^{1}$ found that some cases of RTT were caused by mutations in the MECP2 gene, located on chromosome Xq28. MECP2 encodes the X-linked methyl-CpGbinding protein 2 (MeCP2), which is a global transcriptional repressor and abundant in the brain. MeCP2 specifically binds with its methyl-binding domain (MBD) to symmetrically positioned methylated CpG dinucleotides. Its transcriptional repression domain (TRD) recruits a corepressor complex containing SIN3A and histone deacetylases and results in the repression of the target gene. ${ }^{2}$ Decreased MeCP2 activity will lead to derepression of transcription at multiple CNS loci and overexpression of some genes that may be detrimental to brain development.

\footnotetext{
*Correspondence: Y Shen, Department Biochemistry \& Molecular Biology, Institute of Basic Medical Sciences, CAMS and PUMC, 5, Dong Dan San Tiao, 100005, Beijing, P.R. China. Tel: 8610 65296486, Fax: 8610 6524 0529; E-mail: sheny@cdm.imicams.ac.cn

${ }^{4}$ Both authors contributed equally to this work.

Received 12 February 2002; revised 4 April 2002; accepted 23 April 2002
}

In this study, we report MECP2 mutations in 31 Chinese RTT patients in order to study the spectrum of mutations in Chinese patients.

\section{Materials and methods}

Patients

Thirty-one sporadic cases of classical RTT have been followed up. They were found in nine provinces of China. All of the patients fulfilled the diagnostic criteria proposed by The Rett Syndrome Diagnostic Criteria Work Group. ${ }^{3}$

Genomic DNA was extracted using standard procedures from the peripheral blood leukocytes of patients.

PCR amplification and cloning PCR was performed to amplify all the three exons of MECP2 gene, using published primers and conditions ${ }^{1}$ with some modifications. PCR products were purified and cloned into pGEM $^{\mathbb{R}}$-T vector (Promega). Four random clones of each fragment were selected. The plasmid DNA were extracted and purified.

\section{Sequencing}

The inserts in the plasmids were sequenced with the $\mathrm{ABI}$ 377 DNA sequencer (Perkin-Elmer, Applied Biosystems, CA, USA). Sequencing results were compared with reference 
to the normal human genomic MECP2 sequence and were analysed using the CONSED software. When differences from the normal were found, purified PCR products from these patients were sequenced directly to confirm the original results.

\section{Results}

We found 12 different mutations in the MECP2 gene in 17 of our 31 sporadic RTT patients (about 55\%). The characterized mutations were all in the third exon and were heterozygous. There were four different missense mutations, three different nonsense mutations, five frameshift mutations (Table 1). Two of these are novel: $875 \mathrm{ins} A$ in patient 25 (Figure 1A) and an adjacent double deletion of 26 bp in patient 9 (Figure 1B). In patient 5 , a $G \rightarrow A$ transition at nt 21830 (GenBank accession No. AF030876) was detected. This variant in the $3^{\prime} \mathrm{UTR}$ has been reported previously. ${ }^{4}$ In patient 18 , a $\mathrm{C} \rightarrow \mathrm{G}$ transversion at nucleotide 1141 leads to a novel Proline to Alanine substitution at residue 381 (P381A). Familial analysis in this case indicated that this P381A allele is a benign polymorphism, because the unaffected father carried the same variant.

\section{Discussion}

Codons 168, 255 and 294 are hot spots for truncating mutations. R168X is located upstream of but close to the TRD, but R255X and R294X are within the TRD (Figure 2). Patients 12 and 17, carrying R168X, died at 12 and 4 years of age respectively. Patient 17 was quite unusual. She started to show regression at 5 months of age and deteriorated rapidly, with death at 4 years of age. Patients 4,8 ,

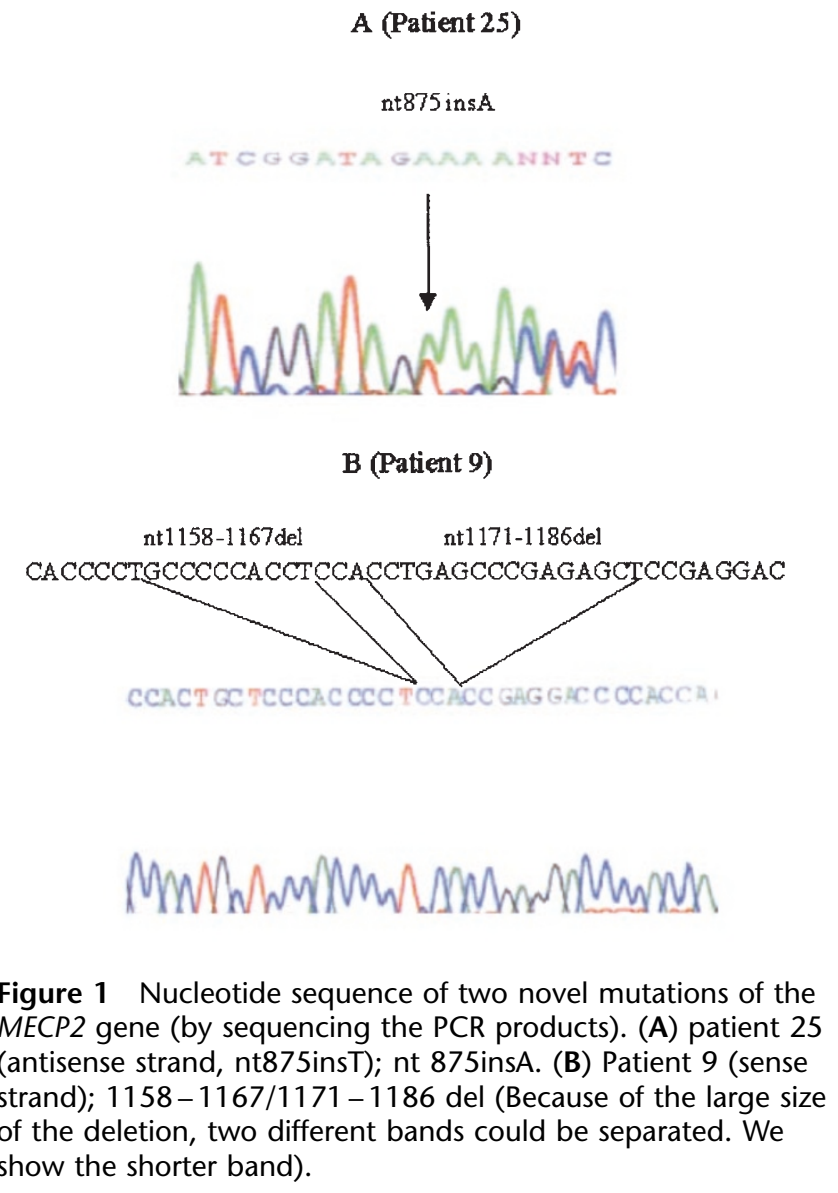

Table 1 Genotype and phenotype of patients carrying MECP2 mutations

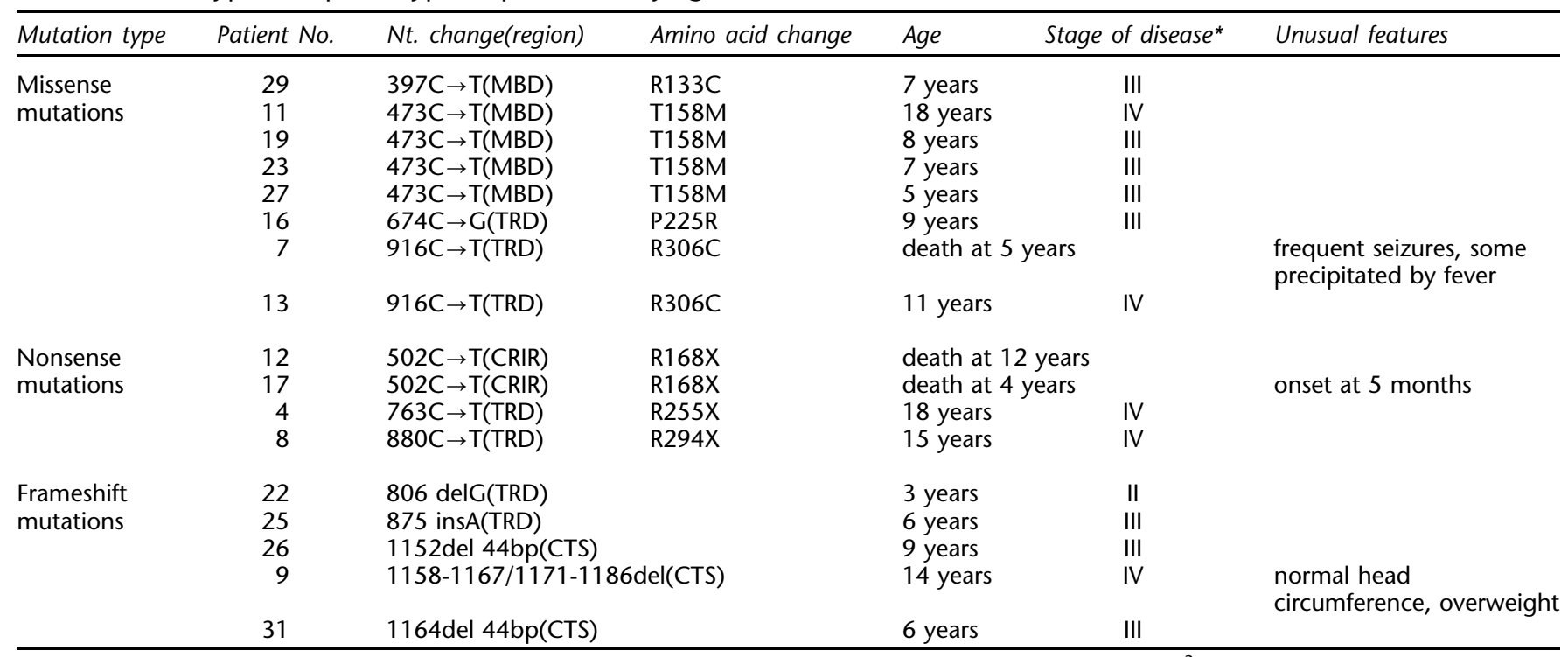

*All patients presented with manifestations required by The Rett Syndrome Diagnostic Criteria Work Group. ${ }^{3}$ For diagnosis; CRIR: Co-repressor
interacting region; CTS: C-terminal segment. 


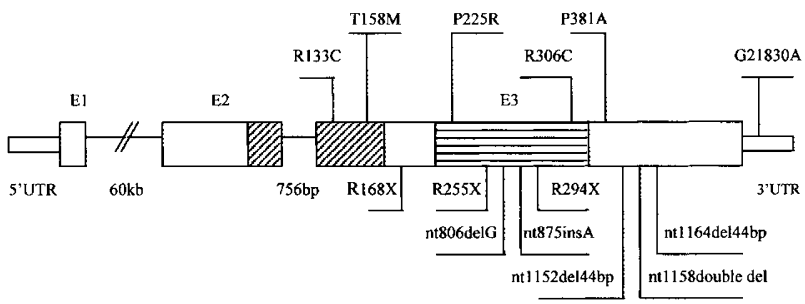

MII

侨R

Figure 2 Distribution of mutations in the MECP2 gene.

9, 22, 25, 26 and 31 carrying either truncating or frameshift mutations were in stage II, III or IV with relatively mild clinical symptoms (Table 1). Patient 9 was examined at 11 years of age. She was only mildly affected. Her head circumference was normal $(54 \mathrm{~cm})$ and she was overweight $(60 \mathrm{~kg}$, height $145 \mathrm{~cm}$ ). So truncating mutations within or downstream of TRD or deletions in the C-terminal region are consistent with reduced clinical severity when compared with truncating mutations N-terminal to this domain. ${ }^{5}$

The large fragment deletions are within a hot spot for deletion or insertion. For the mutation $1152 \mathrm{del} 44 \mathrm{bp}$, both the donor and acceptor sites of the deletion are ACC. For the mutation $1164 \mathrm{del} 44 \mathrm{bp}$, they are GCCCCC. It is of interest to note that all the three large fragment deletions produce premature stop codon at the same position.

A missense variant (P381A) was detected in patient 18 and her unaffected father. Apparently it is non-pathogenic. The daughter's disease could be due to an allelic variant in the non-coding region of MECP2 or a mutation in another gene. Hence, familial studies are important to distinguish polymorphisms from causative mutations.

In our study, direct sequencing of the PCR product frequently gave profiles with a number of background peaks which often interfered with the proper reading of the nucleotide sequence. Hence we first cloned the purified PCR product and sequenced the cloned fragment. However, since we only picked four random clones of each fragment for the initial sequencing, there is a possibility that the clones carried the normal fragment instead of the mutated from the patient who is almost always a heterozygote. This could be the reason for our low mutation pick up rate.

Two novel mutations and a novel benign polymorphism were found. Most of the mutations found are located in mutation hot spots previously reported. It is apparent that recurrent mutations in the MECP2 gene can occur globally. This will help in developing a rapid method of detection/ screening of MECP2 mutations. On the other hand, MECP2 mutations had also been found in non-RTT X-linked mental retardation. ${ }^{6}$ This should be borne in mind in the differential diagnosis of mental retardation in females.

The pathogenesis of RTT remains unclear. Further studies should include more detailed correlation between genotype and phenotype, and the roles of MECP2 gene and MeCP2 protein in neurodevelopment.

\section{Acknowledgments}

We thank Professor Wilson HY Lo for his helpful suggestion and revision of the manuscript. This work was supported by the National Natural Science Foundation of China (Grant 39670459, 39625007 and 39993420), the China National Key Program in Basic Research (G 1998051003), the China National High-Tech R\&D Program (863-102-10-03-05), and the Foundation of Peking University Center for Human Genetic Disease.

\section{References}

1 Amir RE, Van den Veyver IB, Wan M, Tran CQ, Francke U, Zoghbi HY: Rett syndrome is caused by mutations in X-linked MECP2, encoding methly-CpG-binding protein 2. Nat Genet 1999; 23: $185-188$

2 Nan X, Ng HH, Johnson CA et al: Transcriptional repression by the methyl-CpG-binding protein MeCP2 involves a histone deacetylase complex. Nature 1999; 393: 386-389.

3 The Rett Syndrome Diagnostic Criteria Work Group: Diagnostic criteria for Rett syndrome. Ann Neurol 1988; 23: 425-428.

4 Bourdon V, Philippe C, Labrune O, Amsallem D, Arnould C, Jonveaux P: A detailed analysis of the MECP2 gene: prevalence of recurrent mutations and gross DNA rearrangements in Rett syndrome patients. Hum Genet 2001; 108: 43-50.

5 Cheadle JP, Gill H, Fleming $\mathrm{N}$ et al: Long-read sequence analysis of the MECP2 gene in Rett syndrome patients: correlation of disease severity with mutation type and location. Hum Mol Genet 2000; 9: $1119-1129$.

6 Couvert P, Bienvenu T, Aquaviva C et al: MECP2 is highly mutated in X-linked mental retardation. Hum Mol Genet 2001; 10: 941 946. 